\title{
A comparison of pathological methods of measuring lung cancer volume
}

\author{
S Binks, C A Clelland, C Layton
}

\begin{abstract}
Aim-To determine which of several pathological methods of measuring lung cancer volume compared most favourably with the gold standard.

Methods-Three pathological methods were used on 54 resected lung cancers: (1) measuring the maximum dimension and assuming a spherical shape; (2) measuring three dimensions and assuming an ellipsoidal shape; and (3) deriving the volume from the area of tumour on sequential $1 \mathrm{~cm}$ slices using a photocopier and an image analysis system. The gold standard was obtained from the area of whole mount tumour sections on sequential 0.1 cm slices of eight cancers.

Results-Volumes derived from $1 \mathrm{~cm}$ lung slices gave results closest to our gold standard but assuming tumours were ellipsoidal was only a slightly less accurate and less time consuming method. Assuming cancers were spherical resulted in gross overestimation of the tumour volumes.
\end{abstract}

Conclusions-For practical purposes, it is reasonable to measure three dimensions of a lung tumour at sectioning and calculate the volume using the formula for an ellipsoid $(V=4 / 3 \pi$ d.e.f, where $d$, e and f are the semi-axes).

(f Clin Pathol 1996;49:654-656)

Keywords: lung cancer volume.

Primary tumour size is of prognostic importance in many malignancies and this is true for non-small cell carcinoma of the lung (NSCLC). ${ }^{1-3}$ Lung tumour size is an integral part of the American Joint Committee staging system for lung cancer ${ }^{4}$; a T1 tumour is less than $3 \mathrm{~cm}$ in diameter. Tumour volume is the most appropriate measurement of size and with the advent of increasingly sophisticated imaging techniques, accurate in vivo measurements of volume may become feasible. Smaller tumours are easier to resect, less likely to be incompletely excised and also less likely to have metastasised at the time of operation. Kerr et $a \bar{l}$ reported the mean tumour volume of five year survivors as $26 \mathrm{ml}$ compared with $81 \mathrm{ml}$ for non-survivors. Although a useful parameter in NSCLC, to date there is no correlation between small cell lung cancer size and survival. ${ }^{6}$

We have compared three different pathological methods of measuring lung tumour volume with a gold standard method in order to identify the most appropriate method for routine use.

\section{Methods}

Fifty four lung cancers resected between July 1992 and November 1993 from the Depart- $\vec{\omega}$ ments of Thoracic Surgery at the Northern General Hospital, Sheffield, and the City Hos- ? pital, Nottingham, were studied. There was no $\vec{c}$ selection of cases; sequential cases over this time period were studied. There were 32 of squamous carcinomas, 14 adenocarcinomas, three adenosquamous carcinomas, two small cell carcinomas, one bronchioloalveolar cell carcinoma, one mucoepidermoid carcinoma, and one pulmonary blastoma.

At sectioning, the three dimensions of the $\vec{\theta}$ tumours were measured and, using a set up similar to a brain slicing system, the lungs or lobes were cut into $1 \mathrm{~cm}$ slices (fig 1). The slices containing tumour were photocopied (photocopier purchased by the Peel Trust) at a ratio of 1:1 (fig 2) and the boundaries of the $\frac{\bar{D}}{\mathrm{O}}$ tumours were traced onto acetate sheets. The $\stackrel{2}{\Rightarrow}$ tumour area of each slice was obtained using an image analysis system (Seescan). Photocopying of the lung slices was necessary because the image analysis system was located at another hospital. More importantly, the ? image analysis system was unable to distin- $\frac{5}{3}$ guish the tumour boundary accurately from fixed lung slices.

The entire blocks of tumour from eight cases were processed by hand and mounted whole sections of tumour were cut on a sledge micro- $\frac{7}{0}$ tome at $0.1 \mathrm{~cm}$ intervals. The tumour outlines on these whole mount sections were viewed $N$ through a dissecting microscope and traced $N$ onto acetate sheets. The tumour area of each $\underset{\mathrm{N}}{\mathrm{N}}$ slice was obtained using the image analysis system as for the photocopied tumour slices.

The details of the calculation of the four dif- $\frac{\mathbb{D}}{\mathbb{D}}$ ferent volume measurements are as follows:

(1) assuming the tumours to be spherical, $\frac{0}{\circ}$ using the formula $\mathrm{V}=4 / 3(\mathrm{~d} / 2)^{3}$ where $\mathrm{d}=\frac{\mathrm{O}}{\mathbb{D}}$ maximum diameter;

(2) assuming the tumours to be ellipsoidal, ${ }^{7} \stackrel{\mathbb{2}}{2}$ using the formula $V=4 / 3 \pi$ (d.e.f) where $d$, $\mathrm{e}$ ? and $f$ are the semi-axes;

(3) using the areas of tumour slices from photocopied $1 \mathrm{~cm}$ lung slices together with the 흘 number of slices containing tumour (a correc- $\stackrel{?}{?}$ tion was applied to the end slices); and

(4) the gold standard, using the areas calculated from the whole mount sections and the number of sections containing tumour.

The tumours were divided into two groups for further analysis: $<14 \mathrm{ml}$ or $\geqslant 14 \mathrm{ml}$, because 


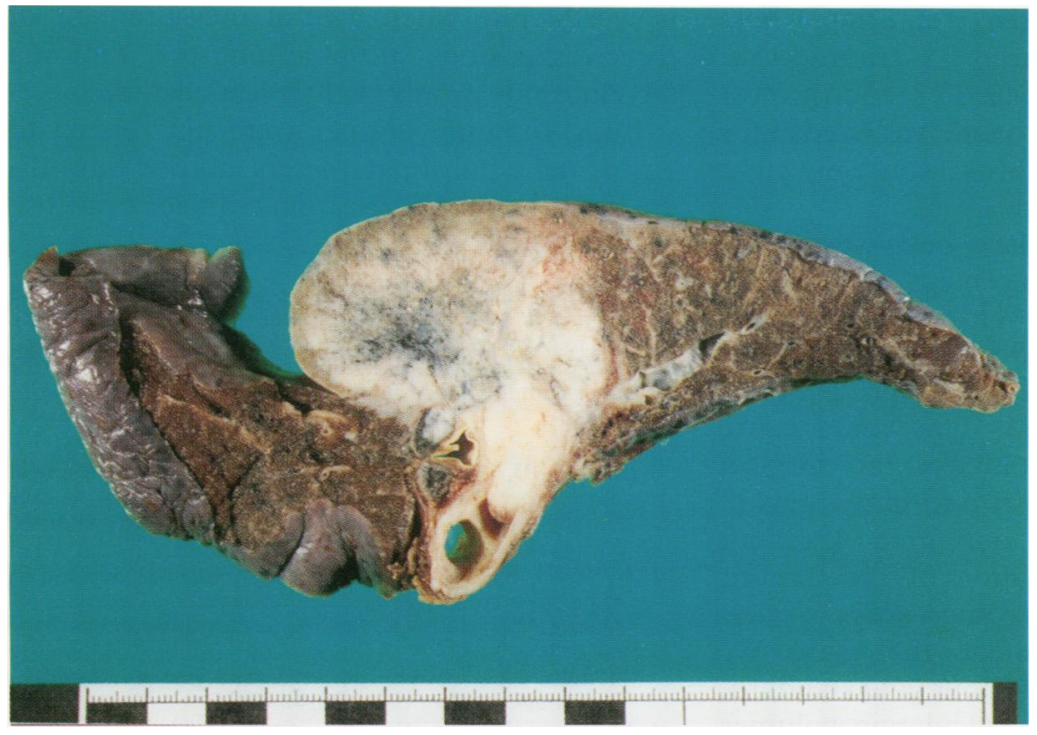

Figure 1 Horizontal $1 \mathrm{~cm}$ section through a central lung cancer demonstrating the bronchial origin and asymmetrical outline. Local lymph node involvement is evident.

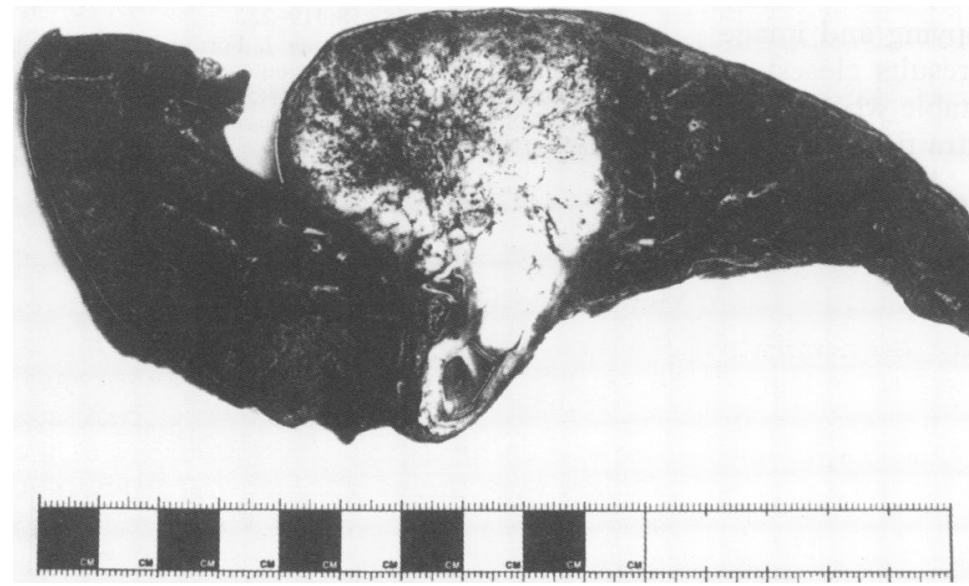

Figure 2 A photocopy of the lung slice in fig 1 shows similar detail, sufficient for the tumour margins to be traced.

a spherical T1 tumour would fit the first category and a T2 tumour the second, assuming there was no involvement of the pleura and no evidence of invasion proximal to a lobar bronchus.

\section{Results}

The range of volumes of the 54 cancers was $0.5-478 \mathrm{ml}$ as spheres, $0.1-224 \mathrm{ml}$ as ellipsoids and $0.2-225 \mathrm{ml}$ using the photocopying method. There was a clear correlation between the gold standard and volumes derived from photocopies of lung slices ( $R=0.932$ ) (fig 3). The correlation between the gold standard and ellipsoid measurements was inferior $(R=$ 0.775), but ellipsoid measurements compared well with the photocopying method $(\mathrm{R}=$ 0.887 ) (fig 4). This was much better than assuming the tumours were spherical; $\mathrm{R}=$ 0.632 when compared with the photocopying method (fig 5). Almost always, the less accurate methods overestimated the tumour volumes.

Lung tumour volumes were not related to the following clinical parameters by $\chi^{2}$ analysis: sex, pathological stage, histological grade, histological type, or presence of metastases. There are insufficient survival data at present for analysis.

\section{Discussion}

The aim of the study was to determine the most suitable method for measuring lung tumour volumes for routine use and to compare various methods with a gold standard. A comparison with values derived from computed tomography will be reported elsewhere. We devised a gold standard method

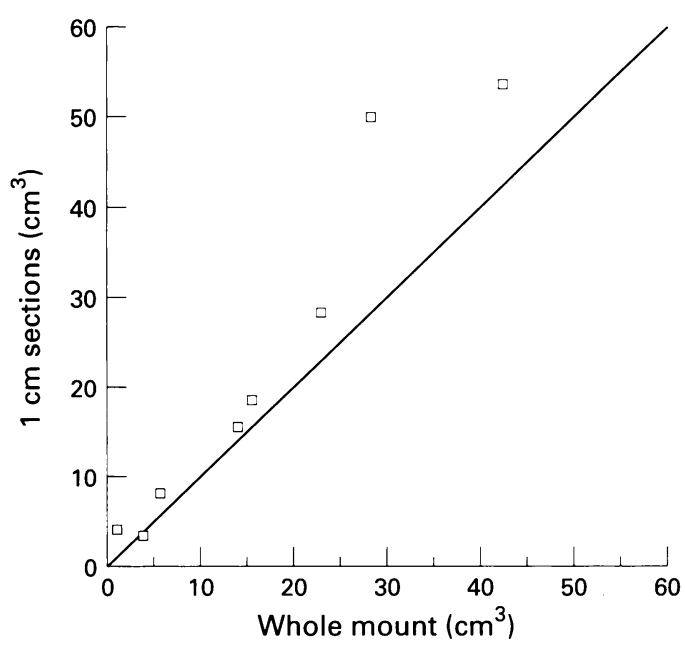

Figure 3 Near linear and 1:1 relation between the gold standard ( $x$ axis) and photocopying method (y axis).

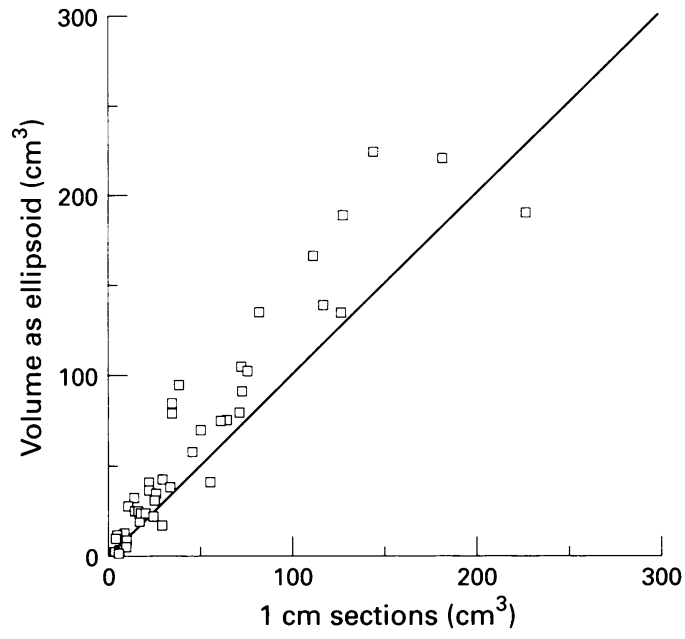

Figure 4 Slight overestimation of ellipsoidal volumes ( $y$ axis) compared with the photocopying method ( $x$ axis).

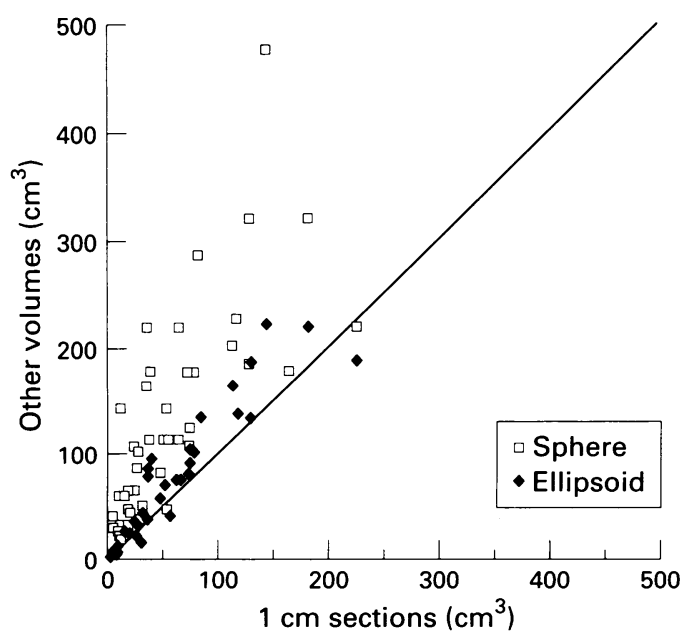

Figure 5 Overestimation of spherical and ellipsoidal volumes ( $y$ axis) compared with the photocopying method ( $x$ axis). 
which was labour intensive, requiring whole mount sections of tumour at $0.1 \mathrm{~cm}$ intervals. Cutting and mounting over 60 sections, most of which were stained by hand, was needed for the larger tumours. For this reason, only eight tumours were treated in this way.

Alternative methods have been suggested, such as the use of displacement of water, to measure tumour volume. With this method the tumour must be dissected from the surrounding stroma and using Archimedes principle the volume is easily obtained. This could cause difficulties in selecting blocks for surgical reporting, especially with regard to margins of excision. Furthermore, our gold standard method permits visualisation of the tumour margin under a dissecting microscope so that atelectasis could not invalidate the results. We therefore have confidence in our gold standard.

Our results show that calculating volumes from a maximum dimension and assuming that lung tumours are spheres is inaccurate and grossly overestimates the volume of many tumours. Using our photocopying and image analysis methods produced results closest to our gold standard. It is debatable whether for routine measurements the extra time required is worthwhile when careful measurement of three dimensions and assuming that tumours are ellipsoids is only slightly less accurate.

Lung tumour volumes were not related to any of the clinical parameters studied but others have shown a correlation with survival. ${ }^{5}$

The authors thank the Peel Trust for the purchase of a photocopier dedicated for pathological specimens. Thanks are 으 also due to Mr W E Morgan, Mr F D Salama and Mr A Thorpe for providing surgical resections and to Trevor Gray for assistance with the image analysis.

1 Jackman RJ, Good CA, Clagett OT, Woolner LB. Survival rates in peripheral bronchogenic carcinomas up to four $\vec{D}$ centimetres in diameter presenting as pulmonary nodules. ๑ § Thorac Cardiovasc Surg 1969;57:1-8.

2 Steele JD, Kleitsch WP, Dunn JE Jr, Buell P. Survival in males with bronchogenic carcinomas resected as asympto- $\vec{O}$ matic solitary nodules. Ann Thorac Surg 1966;2:368-76.

3 Steele JD, Buell P. Asymptomatic solitary pulmonary nodules. Host survival, tumor size, and growth rate. $\mathcal{f}$ Thorac Cardiovasc Surg 1973;65:140-51.

4 American Joint Committee. Staging of lung cancer. $\frac{\overrightarrow{0}}{0}$ Chicago: American Joint Committee for Cancer Staging

5 Kerr KM, Lamb D. A comparison of patient survival and $\infty$ tumour growth kinetics in human bronchogenic carci- of noma. Br f Cancer 1988;58:419-22.

6 Whitley NO, Belani CP, Aisner J, Forrest A, Whitley J. Tumour volume and treatment outcome in small cell lung carcinomas. Invest Radiol 1989;24:581-4.

7 Dunnill MS, Ahere WA. Morphometry. London: Edward Arnold, 1982 